\title{
Procalcitonin and C-Reactive Protein as a Predictor of Organ Dysfunction and Outcome of Sepsis and Septic Shock Patients in Intensive Care Unit
}

\author{
Syafri Kamsul Arif ${ }^{1}$, Maya P. Suyata ${ }^{1}$, Syafruddin Gaus $^{1} \&$ Muhammad Ramli Ahmad ${ }^{1}$ \\ ${ }^{1}$ Department of Anesthesiology, Intensive Care, and Pain Management, Faculty of Medicine, Hasanuddin \\ University, Makassar, Indonesia \\ Correspondence: Syafri Kamsul Arif, Department of Anesthesiology, Intensive Care, and Pain Management, \\ Faculty of Medicine, Hasanuddin University, Makassar, Indonesia. E-mail: syafrikarif@yahoo.com
}

Received: October 1, 2017 Accepted: October 31, 2017 Online Published: November 14, 2017

doi:10.5539/gjhs.v9n12p169 URL: https://doi.org/10.5539/gjhs.v9n12p169

\begin{abstract}
Background and Objective: Sepsis is a potentially life-threatening disorder in ICU. The worst complication is organ dysfunction and mortality. Procalcitonin (PCT) and C-Reactive Protein (CRP) had been proposed as biomarker and predictor for diagnosis, prognosis, and patient deterioration in sepsis and septic shock patients. To know whether PCT and CRP can be used as a predictor of organ dysfunction and outcome in sepsis and septic shock patients in ICU.
\end{abstract}

Materials and Methods: Data were cohort retrospectively analyzed in 35 sepsis (45.5\%) and 42 septic shock patients (54.5\%) admitted to ICU Dr. Wahidin Sudirohusodo General Hospital (January 2014 - December 2015). Data were analyzed using Chi-Square test, Pearson Correlation, and Spearman-Rho Correlation test.

Results: Total of 77 patients met the inclusion criteria. Cut-off point in predicting organ dysfunction in sepsis and septic shock was significantly higher in PCT $(45.7 \mathrm{ng} / \mathrm{mL})$ with $76.6 \%$ sensitivity and $70.0 \%$ specificity, while CRP was $145.75 \mathrm{mg} / \mathrm{mL}$ with $70.2 \%$ sensitivity and $56.7 \%$ specificity. There was a positive correlation of PCT $(0.492[\mathrm{p}=0.000])$ and CRP $(0.336[\mathrm{p}=0.003])$ to organ dysfunction reflected on SOFA score using the Pearson Correlation test $(\mathrm{p}<0.01$ statistically significant). Based on Spearman-Rho Correlation test, correlation of PCT $(0.191[p=0.097])$ and CRP $(0.110[p=0.340])$ to outcome in day-7 was positive but not statistically significant $(\mathrm{p} \geq 0.01)$. While in day-28, there was positive correlation $0.553(\mathrm{p}=0.001)$ for PCT, $0.460(\mathrm{p}=0.006)$ for CRP, and statistically significant $(\mathrm{p}<0.01)$.

Conclusions: Procalcitonin and CRP can be used as a predictor of organ dysfunction and outcome in sepsis and septic shock patients.

Keywords: CRP, procalcitonin, sepsis, outcome, organ dysfunction, septic shock

\section{Significant Statement}

Sepsis is a major cause of mortality and morbidity in critically ill patients. Procalcitonin is a prohormone of calcitonin contained in the human body. In sepsis, increased levels of procalcitonin in the blood has a significant value that can be used as a biomarker of sepsis. Compared with other sepsis biomarkers such as CRP, procalcitonin levels are more sensitive and the most rapid rise after exposure to the infection. In another study, the level of invasion of microorganisms in sepsis provides rhythm correlation with increased levels of procalcitonin blood. Provision of appropriate antibiotic therapy will reduce levels of procalcitonin in sepsis, but antibiotics are not appropriate to raise the levels. The high rate of mortality in sepsis indicates the need for action and research to reduce this mortality, one of them with early diagnosis and management of sepsis. In Indonesia research on the use of the parameters of procalcitonin and CRP in patients with sepsis is still very limited, in Makassar has never done research on the parameters of procalcitonin and CRP in patients with sepsis, therefore, this study aims to determine whether the PCT and CRP can be used as a predictor of organ dysfunction and outcomes of patients with sepsis and septic shock in the ICU.

\section{Introduction}

As a major cause of mortality and morbidity in critically ill patients sepsis is commonly found together with other 
diseases. Both Procalcitonin (PCT) and C-reactive protein (CRP) are biomarkers that most frequently used for sepsis critically ill patients. It is considered to have a higher capacity for diagnosing sepsis patients than CRP. Thus, CRP has been suggested to predict the response of therapeutic and sepsis outcome, result indicated the slower changes have been associated with persistent infection, organ failure, or mortality in the intensive care unit (ICU), (Maraghi, Yehia, Mahmoud, \& AA., 2014). A relatively rapid fall in PCT may be associated with beneficial outcomes of pneumonia, meningitis, and burn-associated, or other infections. Elevated PCT concentrations may be associated with organ failure and mortality and thus might have predictive value (Rajab, 2012).

Sepsis mortality rate is still quite high both in developed countries and in developing countries. Incidence in Europe around 90.4 cases per 100,000 population per year and $28-50 \%$ mortality. In Indonesia have not obtained accurate data about sepsis. The incidence of sepsis in some referral hospitals ranged from 15 to $37.2 \%$ with $37-80 \%$ mortality. Hospital Dr. Cipto Mangunkusumo reported the incidence of sepsis by $25 \%$ with a mortality rate of 77.3\%. (https://aliarsyad.files.wordpress.com/2014/08/journal-ane.doc)

There was several studies about various parameters and assessment or scoring system for the diagnosis, prognosis estimate, assess and monitor the improvement or worsening of sepsis patients. The parameters have been investigated in this case, namely clinical conditions such as temperature, heart rate, blood pressure, and respiration, also some investigations among others the number of leukocytes, protein levels of acute inflammation such as C-reactive protein (CRP), procalcitonin (PCT) and interleukin -6 (IL-6). Clinical parameters as well as the number of leukocytes has been used as a marker of SIRS and sepsis, but the use of other supporting parameters are still hampered by the constraints of the sensitivity, specificity, practicality, and also finance (Singer et al., 2016; Lopez Jimenez, Tobon, Mote, \& Farias, 2011; Joen \& Ji, 2015).

Procalcitonin is a prohormone of calcitonin contained in the human body. In sepsis, increased levels of procalcitonin in the blood has a significant value that can be used as a biomarker of sepsis. Compared with other sepsis biomarkers such as CRP, procalcitonin levels are more sensitive and the most rapid rise after exposure to the infection. In research conducted in preterm infants, age and gender have no bearing significant increase in the levels of procalcitonin in sepsis. PCT is detectable after aninfection following an increase in the levels of pro-inflammatory cytokines such as tumor necrosis factor- $\alpha$ or IL-6, with high levels maintained from 8 to $24 \mathrm{~h}$, after which levels return to baseline values more rapidly than that of CRP. (https://www.ncbi.nlm.nih.gov/pmc/articles/PMC4071182/).

C-Reactive Protein (CRP) is one of the acute phase protein present in normal blood levels will rise when the inflammatory process. CRP has been used as a marker of acute inflammation, produced by the liver in response to tissue damage and infection. CRP levels in plasma have been reported to be associated in parallel with the severity of the infection and sepsis. CRP levels are very sensitive to inflammation that has been widely used as a marker of sepsis. CRP is induced by pro-inflammatory cytokines include Interleukin-1 (IL-1), Interleukin-6 (IL-6), tumor necrosis factor (TNF $\alpha$ ), Interleukin-18 (IL-18) and others. During this CRP is used to distinguish the progression of sepsis, severe sepsis, septic shock and multiple organ failures are higher CRP levels and more severe progression of sepsis. Until now there has been found a marker for the diagnosis of sepsis who have a high accuracy, but experts believe if CRP combined with other markers may be obtained much higher sensitivity. (https://www.chanrejournals.com/index.php/rheumatology/article/view/117/html).

C-reactive protein (CRP) is a well-known biochemical marker of inflammation and has also been shown to be involved in several immunological functions. The usefulness of CRP measurements in the diagnosis of infection has been studied previously in several clinical settings (https://www.chanrejournals.com/index.php/rheumatology/article/view/117/html).

Some studies showed a high sensitivity CRP in diagnosing sepsis (70-80\%) and distinguish between sepsis, severe sepsis, and septic shock. CRP is found as good as procalcitonin in diagnosing sepsis.

Antimicrobial therapy that is promptly administered and controlling the source of infection have been shown to improve outcome in septic patients. If sepsis is well controlled, PCT and CRP may show decreasing patterns. Therefore, changes in biochemical markers may be useful in predicting therapeutic response and prognosis in septic patients. However, the predictive value of these markers is not yet clear (Szederjesi, Almasy, Lazar, Huțanu, Badea, \& Georgescu, 2015). Some studies have shown that it is possible to predict the outcome of sepsis based on changes in PCT and CRP. The aims of this study are to know whether PCT and CRP can be used as a predictor of organ dysfunction and outcome in sepsis and septic shock patients in ICU. 


\section{Materials and Methods}

\subsection{Ethics Statement}

This retrospective study was performed in a cohort of patients with sepsis and septic shock who were admitted to the Intensive Care Unit (ICU) of Wahidin Sudirohusodo General Hospital from January 2014 to December 2015. This study was approved by the Institutional Review Board of Hasanuddin Medical Faculty.

\subsection{Patients and Samples}

We retrospectively analyzed 77 patients, 35 sepsis (45.5\%) and 42 septic shock patients (54.5\%). Adult patients who were older than 18 years and fulfilled the definitions of sepsis or septic shock were recruited. Patients were excluded if they didn't have Procalcitonin and C-Reactive Protein level in first 24 hours, had a history of chemotherapy, ongoing hematologic malignancy disorders, and didn't receive standard supportive treatment following the recommendations of the SSC (Surviving Sepsis Campaign) released in 2012.

\subsection{Statistical Analysis}

Variables are expressed as numbers and percentages, or medians and inter-quartile ranges (IQRs). The predictive performance of each biochemical marker was assessed using the area under the curve (AUC) of receiver operating characteristic (ROC) curves of the sensitivity over1-specificity. AUCs were compared using the nonparametric approach for two correlated AUCs. Correlation of PCT and CRP to organ dysfunction by SOFA were analyzed with Pearson Correlation test. Spearman Rho-correlation method was used to determine the survival curves, which were then compared using log-rank tests for survival data. Both univariate and multiplelogistic regression analysis methods were used to predict the success of a treatment and the 7-day and 28-day mortality. Data were analyzed using the Diagnostic test in SPSS 22.0.

\section{Results}

This study included total 77 cases; 41(53,2\%) males and 36(46,8\%) females. With Chi Square test $p<0.05, p=0.569$ for gender, which is not statistically significant. Average of age in this study was 43.45 (18-65 years old). There was no statistically significant difference with Chi-square test $(\mathrm{p}<0.05)$ in the outcome of sepsis and septic shock, for day-7 $\mathrm{p}=0.569 ; 36$ surviving patients $(46.8 \%)$ and 41 died $(53.2 \%)$. In day-28 $\mathrm{p}=0.170 ; 13$ survived (38.2\%) and 21 dies (61.8). Same result for diagnostic of patient, $\mathrm{p}=0.425$; sepsis 42 patients $(54.5 \%)$ and septic shock 35 patients $(45.5 \%)$. From all of the characteristic, we can conclude that the data were homogeny.

The mean PCT was $75.41 \pm 69,43 \mathrm{ng} / \mathrm{ml}$ (range: 0.05 - $200 \mathrm{ng} / \mathrm{ml}$ ); and CRP $171.42 \pm 79.89 \mathrm{mg} / \mathrm{l}$ (range: 39.40 $437.10 \mathrm{mg} / \mathrm{l}$ ). Cut-off point in predicting organ dysfunction in sepsis and septic shock (Table 1) was significantly higher in PCT $(45.7 \mathrm{ng} / \mathrm{mL})$ with $76.6 \%$ sensitivity and $70.0 \%$ specificity (Figure 1), while CRP was $145.75 \mathrm{mg} / \mathrm{mL}$ with $70.2 \%$ sensitivity and $56.7 \%$ specificity (Figure 2 ).

Table 1. Comparison sensitivity and specificity of PCT and CRP in predicting organ dysfunction in sepsis and septic shock patients

\begin{tabular}{lllllll}
\hline Variable & Cut off point & $\begin{array}{l}\text { Sensitivity } \\
(\%)\end{array}$ & $\begin{array}{l}\text { Spesificity } \\
(\%)\end{array}$ & $\begin{array}{l}\text { PPV } \\
(\%)\end{array}$ & $\begin{array}{l}\text { NPV } \\
(\%)\end{array}$ & $\begin{array}{l}\text { Accuracy } \\
(\%)\end{array}$ \\
\hline PCT $(\mathrm{ng} / \mathrm{m} \mathrm{L})$ & 45.7 & 76.6 & 70.0 & 80.0 & 65.6 & 70.4 \\
CRP $(\mathrm{mg} / \mathrm{L})$ & 145.75 & 70.2 & 56.7 & 71.7 & 54.8 & 64.9 \\
\hline
\end{tabular}


$\mathrm{AUC}=\mathbf{0 , 7 6 5}$

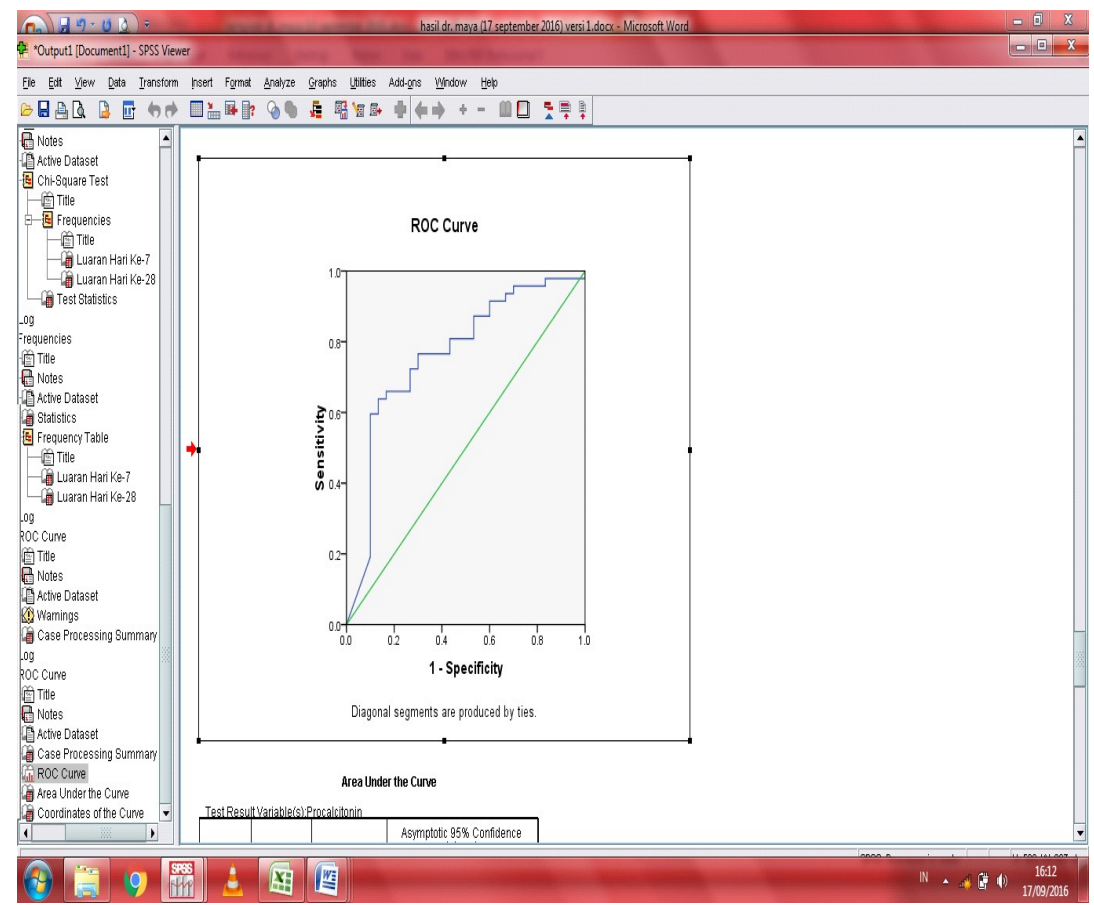

Figure 1. ROC for sepsis patients with organ dysfunction based on PCT

$\mathrm{AUC}=\mathbf{0 , 6 8 7}$

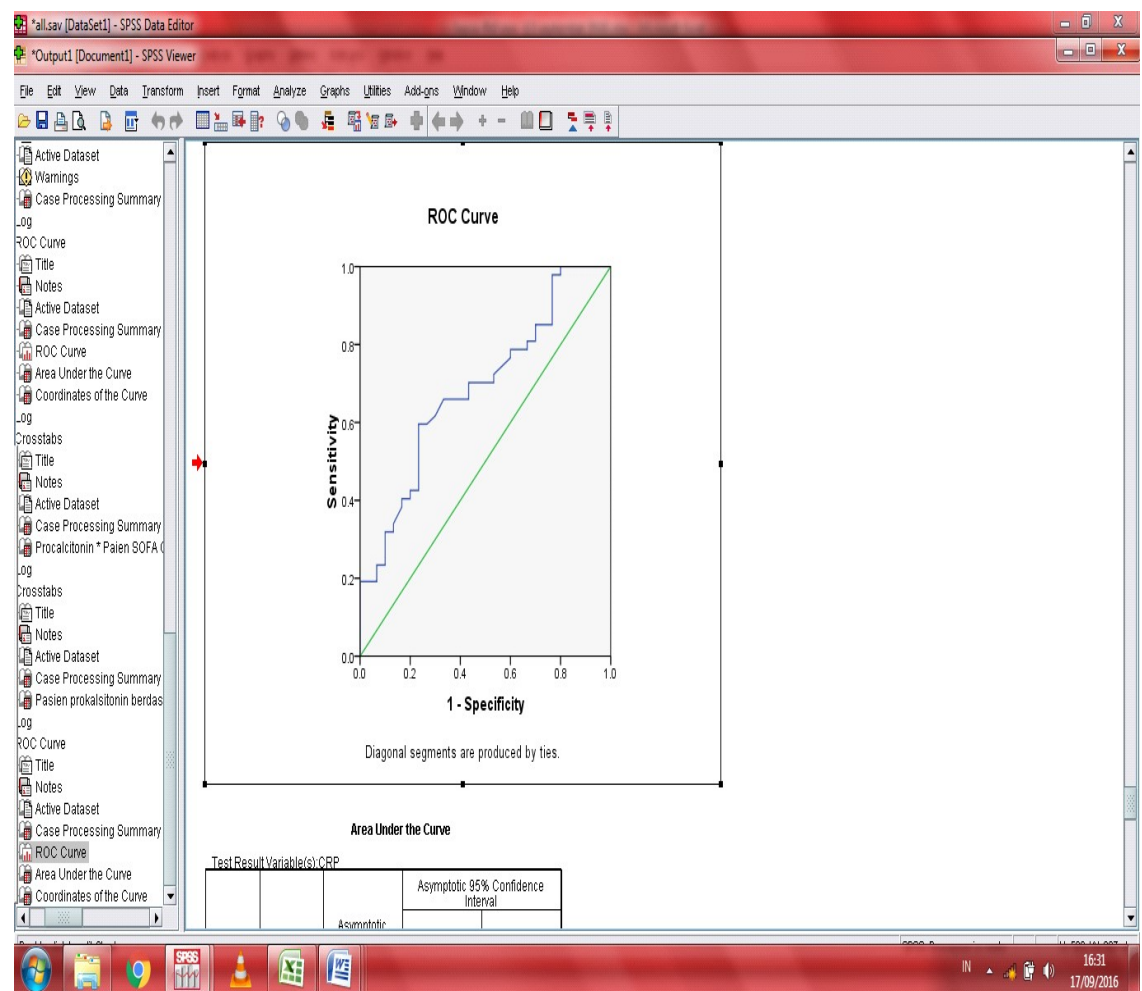

Figure 2. ROC for sepsis patients with organ dysfunction based on CRP

There was a positive correlation of PCT $(0.492[\mathrm{p}=0.000])$ and CRP $(0.336[\mathrm{p}=0.003])$ to organ dysfunction reflected on SOFA score using the Pearson Correlation test $(\mathrm{p}<0.01$ statistically significant). (Table 2$)$ 
Table 2. Correlation of PCT and CRP to SOFA score

\begin{tabular}{lll}
\hline \multirow{2}{*}{ Variable } & \multicolumn{2}{l}{ SOFA score } \\
\cline { 2 - 3 } & $r$ & $p^{* *}$ \\
\hline PCT $(\mathrm{ng} / \mathrm{mL})$ & 0.492 & 0.000 \\
CRP $(\mathrm{mg} / \mathrm{L})$ & 0.336 & 0.003 \\
\hline
\end{tabular}

$* * p<0.01$ statistically significant, analyzed by Pearson correlation test.

$r$ : correlation value.

Based on Spearman-Rho Correlation test, correlation of PCT $(0.191[\mathrm{p}=0.097])$ and CRP $(0.110$ [p=0.340]) to outcome in day-7 was positive but not statistically significant $(\mathrm{p} \geq 0.01)$. While in day-28, there was positive correlation $0.553(\mathrm{p}=0.001)$ for PCT, $0.460(\mathrm{p}=0.006)$ for CRP, and statistically significant $(\mathrm{p}<0.01)$. (Table 3)

Table 3. Correlation of PCT and CRP to outcome

\begin{tabular}{llllll}
\hline \multirow{2}{*}{ Variable } & \multicolumn{2}{l}{ Outcome Day-7 } & & \multicolumn{2}{l}{ Outcome Day-28 } \\
\cline { 2 - 3 } \cline { 5 - 6 } & $r$ & $p^{* *}$ & & \multicolumn{2}{c}{$p^{* *}$} \\
\hline PCT (ng/mL) & 0.191 & 0.097 & & 0.553 & 0.001 \\
CRP (mg/L) & 0.110 & 0.340 & 0.460 & 0.006 \\
\hline
\end{tabular}

$* * p<0.01$ statistically significant, analyzed by Spearman-Rho correlation test.

$r$ : correlation value.

\section{Discussion}

Data are lacking on the precise role of PCT and CRP in diagnosing septic shock and for their potential use as a prognostic indicator in cases of the sepsis and septic shock. There was no significant difference of mean serum PCT and CRP values between those cases with sepsis and septic shock. This is in agreement with results of other studies.

In this study showed that both of PCT and CRP can be used to predict organ dysfunction in sepsis and septic shock with AUC 0.765 for PCT (cut off point $45.7 \mathrm{ng} / \mathrm{ml}$ ) and AUC 0.687 for CRP (cut off point $145.75 \mathrm{mg} / \mathrm{l}$ ). PCT had a better sensitivity and specificity.

PCT was first described as a marker of the extent and course of systemic inflammatory response to bacterial and fungal infections. Ever since then Procalcitonin (PCT) has been examined extensively as a marker for systemic inflammation, infection, and sepsis, both singularly and in combination with other markers such as CRP, in adults and children in ICU setup (Carr, 2015).

CRP is a traditional marker of sepsis. In 1930, Tillet and Francis identified the capacity to precipitate polysaccharide fractions, designated fraction $\mathrm{C}$, from Streptococcus pneumonia in serum from patients with pneumonia. CRP is the most frequently used biomarker in clinical practice. Previous studies have shown daily CRP measurements are useful for monitoring the course of sepsis in critically ill patients and may be used to indicate successful treatment. CRP monitoring represents a possible means of stopping antibiotics safely, sparing patients from drug toxicity likely decreasing the risk of resistance and decreasing costs. Normalization of CRP concentrations has been proposed as a guideline for stopping antibiotics. In addition, CRP is an inexpensive, consistent, and reproducible test that is available in most hospitals (Joen \& Ji, 2015).

Pro-calcitonin had a positive correlation and statistically significant with Pearson correlation test $(p<0.01)$, the higher value of PCT followed by the higher increase in SOFA score, this relationship had good strength with value of correlation $\mathrm{r}=0.492$. Likewise, CRP also had a significant correlation with SOFA score and the relationship had a good strength too with value of correlation $\mathrm{r}=0.336$. Therefore, both can be used as a predictor of organ dysfunction as a complication of sepsis and septic shock.

Rajab in (Rajab, 2012) concluded the existence of a positive correlation between levels of lactate and CRP with multiple organ dysfunction. This study used a parameter of SOFA score to assess the degree of organ dysfunction. Until today there is no perfect biomarker each has limitations. Therefore, the clinician's role is very important in 
making a decision, but at least with the help of biomarkers supported by good clinical examination and laboratory tests and other supporting the management of sepsis infection will get better.

Based on data analysis using the Spearman-Rho, it can be seen that the relationship procalcitonin levels on the 7th day outcomes for patients with sepsis and septic shock, there was a positive correlation (0.191) but not significant $(\mathrm{p} \geq 0.01)$. This means that the higher the levels of procalcitonin, the more likely the person died on the 7th day, or conversely the lower levels of procalcitonin, the lower is also the possibility the person died on the $7^{\text {th }}$ day, although not significantly. While the association of CRP levels on the 7th day outcomes for patients with sepsis and septic shock, there is also a positive correlation $(0.110)$ and also not significant $(p \geq 0.01)$. This means that the higher the levels of CRP, the more likely the person died on the 7th day, or conversely the lower CRP levels, the lower is also the possibility the person died on the 7th day, although not significantly. Therefore, both can be used as a predictor of patient outcome 7 th and 28th days but more significantly on day 28 .

Procalcitonin levels to the outcome of the day 28 , there was a positive correlation $(r=0.553)$ and statistically significant $(\mathrm{p}<0.01)$. This means that the higher the levels of procalcitonin, the more likely the person died on day 28 , or conversely the lower levels of procalcitonin, the lower is also the possibility the person died on day 28 , significantly. While the association of CRP levels on the outcome day 28 for patients with sepsis and septic shock, there was also a positive correlation $(\mathrm{r}=0.460)$ and significant $(\mathrm{p}<0.01)$. This means that the higher the levels of CRP, the more likely the person died on day 28 , or conversely the lower CRP levels, the lower is also the possibility the person died on day 28 , significantly.

(Nargis, Ibrahim, \& Ahamed, 2014) concluded that procalcitonin is better than CRP to identify and assess the severity of sepsis although both cannot be used to distinguish between infectious and not aninfection. Joen et al (Joen \& Ji, 2015) concluded that the superior procalcitonin as a marker of early sepsis compared with CRP.

(Ryu et al., 2015) performed a retrospective study in patients with severe sepsis and septic shock was admitted to the ICU the results showed that the numbers procalcitonin and CRP was not associated with death but rather related to the success of therapy in sepsis patients. Additionally in this study noted that procalcitonin not better than CRP in predicting outcomes in sepsis patients treated in the ICU.

The high rate of mortality in sepsis indicates the need for action and research to reduce this mortality, one of them with early diagnosis and management of sepsis. In Indonesia research on the use of the parameters of procalcitonin and CRP in patients with sepsis is still very limited, in Makassar has never done research on the parameters of procalcitonin and CRP in patients with sepsis, therefore this research becomes important to know the effective management of sepsis patients (Lopez et al., 2011; Shehabi et al., 2014).

\section{Conclusions}

Procalcitonin and C-Reactive Protein can be used as a predictor of organ dysfunction and long-term outcome in sepsis and septic shock patients. Both of them have a positive correlation with organ dysfunction and outcome.

\section{Conflict of Interest}

All authors declare that there is no any conflict of interest within this research, including in funding support.

\section{Authors Contribution}

- Syafri Kamsul Arif contributed: in study design, data analysis, and manuscript preparation

- Maya Permatasari Suyatacontributed in data collecting and data analysis

- Syafruddin Gauss contributedto data analysis and manuscript preparation

- Muhammad Ramli Ahmad contributed in discussion development and references collection

\section{Acknowledgements}

In addition to the authors, the following persons have served as special editorial consultants and translators for this journal. We wish to take this opportunity to extend our grateful appreciation to all whose who have assisted in the peer review manuscripts submitted to this journal.

Andi Husni Tanra

Ilham Jaya Patellongi

Sitti Wahyuni

Hisbullah

Faisal Muchtar 
Haizah Nurdin

Ari Santri Palinrungi

Andi Adil

Raina Diah Grahani

Andi Bau Sumange Rukka

\section{Competing Interests Statement}

The authors declare that they has no competing or potential conflicts of interest.

\section{References}

Carr, J. A. (2015). Procalcitonin-guided antibiotic therapy for septic patients in the surgical intensive care unit. Journal of intensive care, 3(1), 36. https://doi.org/10.1186/s40560-015-0100-9

https://aliarsyad.files.wordpress.com/2014/08/journal-ane.doc.

https://www.chanrejournals.com/index.php/rheumatology/article/view/117/html.

https://www.ncbi.nlm.nih.gov/pmc/articles/PMC4071182/.

Joen, J. S., \& Ji, S. M. (2015). Diagnostic value of procalcitonin and CRP in critically ill patients admitted with suspected sepsis. Journal of Dental Anesthesia and Pain Medicine, 15(3), 135-140.

Lopez, F. R. E., Jimenez, A. E. R., Tobon, G. A. C., Mote, J. U. D., \& Farias, O. N. (2011). Procalcitonin (PCT), C reactive protein (CRP) and its correlation with severity in early sepsis. Clinical Reviews and Opinions, 3(3), 26-31.

Maraghi, S. E., Yehia, A., Mahmoud, O., \& AA., H. (2014). Procalcitonin versus C-reactive protein at different SOFA scores in ICU Sepsis. Med. J. Cairo Univ, 82(1), 29-36.

Nargis, W., Ibrahim, M., \& Ahamed, B. U. (2014). Procalcitonin versus C-reactive protein: Usefulness as biomarker of sepsis in ICU patient. International journal of critical illness and injury science, 4(3), 195.

Rajab. (2012). Hubungan kadar laktat dengan disfungsi organ multipel pada pasien sepsis di Makassar (Tesis). Makassar: Universitas Hasanuddin.

Ryu, J. A., Yang, J. H., Lee, D., Park, C. M., Suh, G. Y., Jeon, K., ... \& Chung, C. R. (2015). Clinical usefulness of procalcitonin and C-reactive protein as outcome predictors in critically ill patients with severe sepsis and septic shock. PloS one, 10(9), e0138150. https://doi.org/10.1371/journal.pone.0138150

Shehabi, Y., Sterba, M., Garrett, P. M., Rachakonda, K. S., Stephens, D., Harrigan, P., ... \& Ding, G. (2014). Procalcitonin algorithm in critically ill adults with undifferentiated infection or suspected sepsis. A randomized controlled trial. American journal of respiratory and critical care medicine, 190(10), 1102-1110.

Singer, M., Deutschman, C. S., Seymour, C. W., Shankar-Hari, M., Annane, D., Bauer, M., ... \& Hotchkiss, R. S. (2016). The third international consensus definitions for sepsis and septic shock (sepsis-3). Jama, 315(8), 801-810

Szederjesi, J., Almasy, E., Lazar, A., Huţanu, A., Badea, I., \& Georgescu, A. (2015). An evaluation of serum procalcitonin and C-reactive protein levels as diagnostic and prognostic biomarkers of severe sepsis. The Journal of Critical Care Medicine, 1(4), 147-153.

\section{Copyrights}

Copyright for this article is retained by the author(s), with first publication rights granted to the journal.

This is an open-access article distributed under the terms and conditions of the Creative Commons Attribution license (http://creativecommons.org/licenses/by/4.0/). 